\title{
A Teaching Resource for a Computational Framework of the Value of Information in Origin of Life Questions
}

\author{
Soumya Banerjee ${ }^{1,2,3}$, Joyeeta Ghose ${ }^{1}$
}

\author{
${ }^{1}$ University of Oxford \\ Oxford, UK \\ ${ }^{2}$ Ronin Institute \\ Montclair, USA \\ ${ }^{3}$ Complex Biological Systems Alliance \\ North Andover, USA
}

\begin{abstract}
Information plays a critical role in complex biological systems. Complex systems like immune systems and ant colonies co-ordinate heterogeneous components in a decentralized fashion. How do these distributed decentralized systems function? One key component is how these complex systems efficiently process information. These complex systems have an architecture for integrating and processing information coming in from various sources and points to the value of information in the functioning of different complex biological systems. This paper is a teaching resource that explains the role of information processing in questions around the origin of life and suggests how computational simulations may yield insights into questions related to the origin of life.
\end{abstract}

A computational model of the origin of life would unify thermodynamics with information processing and we would gain an appreciation of why proteins and nucleotides evolved as the substrate of computation and information processing in living systems that we see on Earth. Answers to questions like these may give us insights into non-carbon based forms of life that we could search for outside Earth.

We hypothesize that carbon-based life forms are only one amongst a continuum of life-like systems in the universe. Investigations into the role of computational substrates that allow information processing is important and could yield insights into: 1) novel non-carbon based computational substrates that may have "life-like" properties, and 2) how life may have actually originated from non-life on Earth. Life may exist as a continuum between non-life and life and we may have to revise our notion of life and how common it is in the universe. Looking at life or life-like phenomenon through the lens of information theory may yield a broader view of life. 


\section{Teaching Resource}

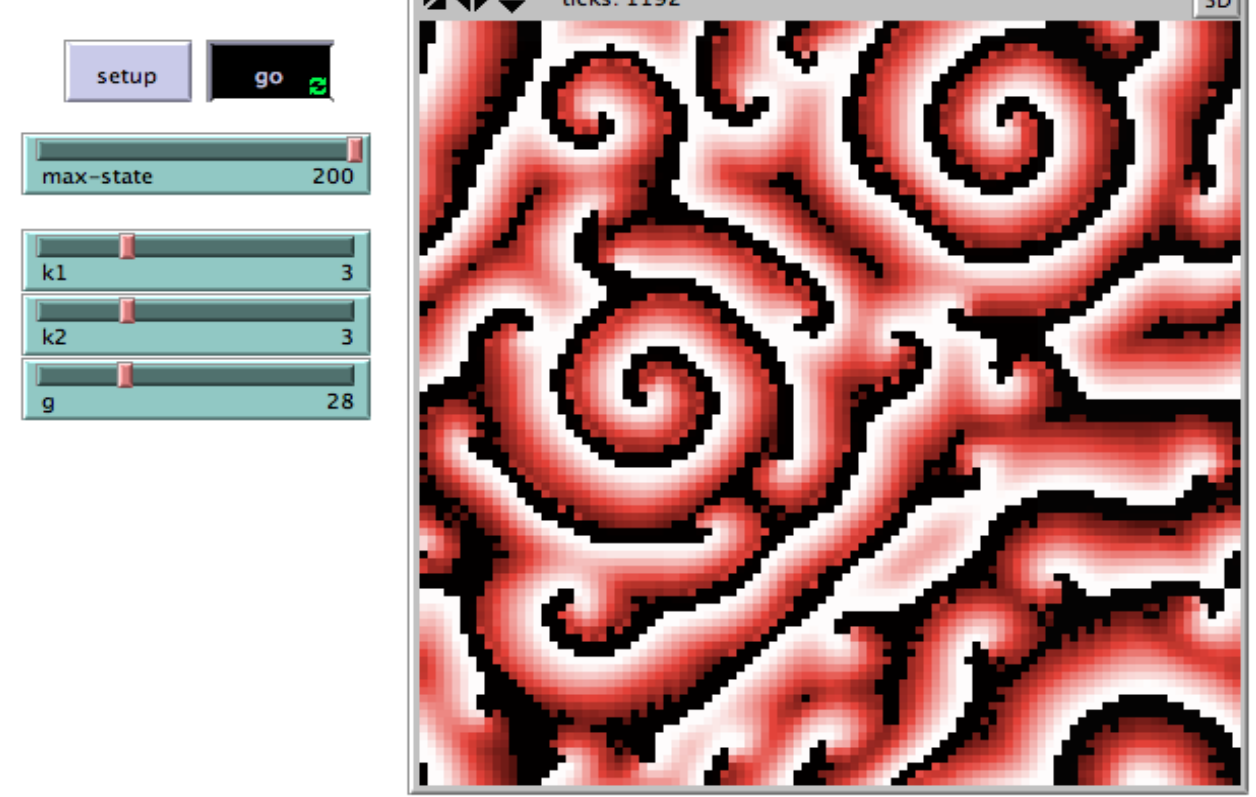

Figure 1. Screenshot from the NetLOGO simulation tool for the Belousov-Zhabotinsky (B-Z) reaction showing wave like patterns that persist $[20,21]$

This teaching resource is for high-school and undergraduate students. We assume that the students will have a basic background and interest in science.

Our conception of life is shaped by what we see around us on Earth. What life forms might we expect to see on alien planets? Would they be carbon-based like us or can they be even more exotic? Answering questions like these mean we have to come up with an objective definition of life.

Chemical reaction systems called reaction-diffusion systems have been studied for their complex properties for a long time. One example is the Belousov-Zhabotinsky (B-Z) reaction which is a chemical oscillator and displays complex properties reminiscent of life (Fig. 1) $[1,2]$. The full model is available on the NetLOGO platform [2]. Reaction-diffusion systems have been exploited to construct computational systems called "reaction-diffusion computers" $[3,4]$.

We have previously hypothesized that an objective definition of life is that it should be capable of information processing [5]. Here we outline activities that would familiarize students and lay audiences with these concepts. 


\section{Learning Activities}

1) Warm-up questions.

a. How might one define life?

b. How might we recognize life that is completely "alien" to us?

c. Does life need to be carbon-based?

d. How do movies bias our conception of alien life-forms (do they have to be little green men?)

e. How would you recognize life if it does not fit the definition of life we have seen on Earth?

2) Demonstration of software. Download the NetLOGO software [1] and experiment with the Belousov-Zhabotinsky (B-Z) model. For what parameters do you observe emergence of "interesting" patterns?

a. Is this life-like?

b. Would you call this life if you saw it on another planet?

c. Peer-discussion and take feedback.

3) Read the papers on reaction-diffusion systems and understand how they have been used to construct computational systems called "reaction-diffusion computers" $[3,4]$.

4) Read the paper on a computational theory of the value of information in origin of life questions [5].

a. Discussion. What did you learn from these papers?

5) Register on SAGANet (www.SAGANet.org) to join a community of people interested in questions around origins of life and astrobiology. Contribute to a discussion forum on SAGANet.

6) Have a discussion on what is life and how might you recognize it if you were to find it in another part of the Universe.

7) Writing task and group presentation.

\section{Evaluation}

Here we present a rubric for how the class performance can be evaluated by the instructor or peers.

1. Delivery of presentation

a. Was the presentation on topic?

b. Were the main ideas clearly communicated?

2. Organization and format of writeup

a. Does the writeup have a good introduction?

b. Is it properly formatted?

c. Are there any grammatical errors? 
3. Originality of content

a. Was the content original?

b. Did the students make an effort to develop new ideas?

4. Analysis of literature review

a. Did the group assimilate the findings of the background reading into the writeup and presentation?

\section{References}

[1] Wilensky, U. (1999). NetLogo. http://ccl.northwestern.edu/netlogo/. Center for Connected Learning and Computer-Based Modeling, Northwestern University, Evanston, IL.

[2] Wilensky, U. (2003). NetLogo B-Z Reaction model.

http://ccl.northwestern.edu/netlogo/models/B-ZReaction. Center for Connected Learning and Computer-Based Modeling, Northwestern University, Evanston, IL.

[3] Adamatzky, Andrew and De Lacy Costello, Benjamin.Experimental logical gates in a reaction-diffusion medium: The XOR gate and beyond. Physical Review E, 4, 66, 2002

[4] Adamatzky, Andrew and de Lacy Costello, Benjamin and Ratcliffe, Norman M., Experimental reaction-diffusion pre-processor for shape recognition, Physics Letters, 344$352,297,2002$

[5] Banerjee, Soumya. A Roadmap for a Computational Theory of the Value of Information in Origin of Life Questions, Interdisciplinary Description of Complex Systems, 14(3), 2016 